\title{
Split: a flexible and efficient algorithm to vector-descriptor product ${ }^{*}$
}

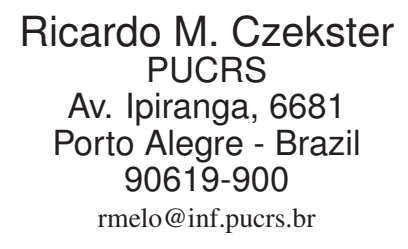

\author{
Paulo Fernandes \\ PUCRS-CNPq \\ Av. Ipiranga, 6681 \\ Porto Alegre - Brazil \\ 90619-900 \\ paulo.fernandes@pucrs.br
}

\author{
Jean-Marc Vincent \\ Laboratoire LIG \\ Project MESCAL \\ 51, Av. Jean Kuntzmann \\ 38330 Montbonnot, France \\ Jean-Marc.Vincent@imag.fr
}

\author{
Thais Webber $^{\dagger}$ \\ PUCRS \\ Av. Ipiranga, 6681 \\ Porto Alegre - Brazil \\ 90619-900 \\ twebber@inf.pucrs.br
}

\begin{abstract}
Many Markovian stochastic structured modeling formalisms like Petri nets, automata networks and process algebra represent the infinitesimal generator of the underlying Markov chain as a descriptor instead of a traditional sparse matrix. A descriptor is a compact and structured storage based on a sum of tensor (Kronecker) products of small matrices that can be handled by many algorithms allowing affordable stationary and transient solutions even for very large Markovian models. One of the most efficient algorithms used to compute iterative solutions of descriptors is the Shuffle algorithm which is used to perform the multiplication by a probability vector. In this paper we propose an alternative algorithm called Split, since it offers a flexible solution between the pure sparse matrix approach and the Shuffle algorithm using a hybrid solution. The Split algorithm puts the Shuffle approach in perspective by presenting a faster execution time for many cases and at least the same efficiency for the worst cases. The Split algorithm is applied to solve two SAN models based on real problems showing the practical contribution of this paper.
\end{abstract}

\section{Keywords}

Performance Evaluation, Numerical Methods, Tensor Algebra, Kronecker Products

\footnotetext{
${ }^{*}$ This work is partially supported by the brazilian government (CAPES), FINEP project STGSD grant nb. 4284/05 and SMS Project (France). Authors Ricardo M. Czekster and Thais Webber are invited by the Project MESCAL-INRIA.

${ }^{\dagger}$ Corresponding author. The order of authors is merely alphabetical.
}

Permission to make digital or hard copies of all or part of this work for personal or classroom use is granted without fee provided that copies are not made or distributed for profit or commercial advantage and that copies bear this notice and the full citation on the first page. To copy otherwise, to republish, to post on servers or to redistribute to lists, requires prior specific permission and/or a fee.

SMCtools '07, October 26, 2007, Nantes, France

Copyright 2007 ICST 978-963-9799-00-4.

\section{INTRODUCTION}

Modeling large complex systems always requires a structured description. The state space explosion itself exists only because we can describe a very large system, i.e., a system with a huge state space, by describing a structure simple enough to be understood by us, humans. Therefore, it is admissible to rely on a structured description to all problems sufficiently large to be called that way. This is evident observing a stochastic Petri net (SPN) [1] or a stochastic automata network (SAN) [24], and even when you look deeply how large straightforward Markov chains are defined, e.g., the balls and buckets concept in MARCA [25]. Using a less procedural approach, process algebras [19] or graph grammars [14] also use a structured, and very modular, description.

One interesting option to keep the structured characteristics of a continuous-time Markovian model into its internal representation is to employ tensor (Kronecker) algebra to store the infinitesimal generator of a given model. The basic principle that recommends the use of tensor representation for infinitesimal generators is to take advantage of all the structural information already used in the original description of the model. Such principle appears since the first definitions of stochastic automata networks [24], but recently it has also been used in other stochastic formalisms [12, 19]. In all those references, the term descriptor is used to refer to a tensor represented infinitesimal generator. The reader interested in detailed information about tensor algebra can found basic concepts of Classical and Generalized Tensor Algebra in [2, 11, 16].

The use of tensor representations for the infinitesimal generator, however, is not always helpful. It undeniably reduces the memory requirements $[22,5]$, but it often increases the CPU time needed to achieve stationary or transient solution. One of the major problems with structured representations is the insertion of unreachable states, but to cope with that, very efficient approaches deal with the determination of reachable set $[10,23]$. It remains an open problem the efficient solution of large and complex models where all, or almost all, states are reachable.

The numerical algorithms known to compute the exact solution of large reachable state space, non-product form, Kronecker represented models are usually iterative and they are based on the vector-descriptor product. This operation can be done by using clever data structures, e.g., matrix diagrams [21], or using a sum 
of tensor product of standard matrix structures [16].

Despite the algorithmic differences, both approaches can be summarized in finding an efficient way to multiply a (usually huge) vector by a non-trivial structure. Old stochastic Petri net solutions [1] translate the model representation into a singular sparse matrix. Obviously, this sparse approach cannot be employed to deal with really large models (e.g.more than 500 thousand states), since it usually requires the storage of a too large sparse matrix (e.g.more than 4 million nonzero elements). The usual stochastic automata network (SAN) solution, called Shuffle algorithm, deals with permutations of matrices and tensors [16] and it can be applied to virtually any structured model. Unfortunately, the Shuffle algorithm suffers with a high CPU cost for many practical cases.

The challenging problem for the numerical solution of structured huge Markov models is to speedup the basic operation of most iterative solutions, the vector-descriptor product. This operation corresponds to the product of a probability vector $v$, as big as the product state space, by a descriptor $Q$. The structure of $Q$ is the ordinary sum of $N+2 E$ tensor products of $N$ matrices, where $N$ is the number of automata, and $E$ is the number of synchronizing events in the model. Therefore, by a simple distributive property, vectordescriptor product algorithms can be viewed in a simpler format as a sum of products of the a vector $v$ by a tensor product term composed by $N$ matrices:

$$
\sum_{j=1}^{N+2 E}\left(v \times\left[\bigotimes_{i=1}^{N} Q_{j}^{(i)}\right]\right)
$$

The reader interested in further details about the descriptor structure can find extensive material in [15].

There are many possible approaches to cope with such problems, varying from hybrid solutions between simulation and numerical analysis [7] to alternative storage structures [8, 23]. The focus on this paper proposition is on pure numerical manipulation bringing together the advantages of a straightforward sparse matrix approach [26] and the Shuffle algorithm [16]. A different solution, called Slice algorithm, was proposed in [17] where a nearly sparse technique was applied. One of the main contributions of the Split algorithm to this domain is the introduction of a flexible approach, since it allows a tailored solution to each tensor product term that may be more time efficient and less memory demanding than any other methods. In fact, the extreme options of the Split algorithm are at least as time efficient as the sparse approach, or as memoryefficient as the Shuffle algorithm.

This paper is organized as follows. Section 2 present the pure sparse algorithm which is theoretically a time-efficient approach. Section 3 presents the Shuffle algorithm which is the memoryefficient approach. Section 4 presents the intermediate Slice algorithm in order to introduce the central contribution of this paper. Section 5 presents the core of this paper which is the Split algorithm and few variants. Section 6 presents numerical results comparing the application of the Split algorithm of some families of SAN models. Finally, the conclusion emphasizes this paper contributions and future works.

\section{TIME-EFFICIENT ALGORITHM}

The more intuitive method to solve the problem, called Sparse algorithm, aims to consider the tensor product term as a unique sparse matrix, multiplying it by a product space sized probability vector. Considering a tensor product term of $N$ matrices $Q^{(i)}$, each one of order $n_{i}$, and with $n z_{i}$ nonzero elements, the Sparse algorithm generates element by element one large matrix $Q$ resulting of $\otimes_{i=1}^{N} Q^{(i)}$. Then the corresponding elements of vector $v$, also with size $\prod_{i=1}^{N} n_{i}$, are multiplied using a traditional algorithm to the vector-sparse matrix product.

Defining $\theta(1 \ldots N)$ as the set of all possible combinations of nonzero elements of the matrices from $Q^{(1)}$ to $Q^{(N)}$, the cardinality of $\theta(1 \ldots N)$, and consequently the number of nonzero elements in $Q$, is given by: $\prod_{i=1}^{N} n z_{i}$. Additionally, the Sparse algorithm needs the information of the size of the state space corresponding to all matrices after the $k$-th matrix of the tensor product, called $n$ right $t_{k}$, and numerically defined by $\prod_{i=k+1}^{N} n_{i}$. Further in this paper, it will be also used the analogous concept of nleft which is numerically equal to $\prod_{i=1}^{k-1} n_{i}$.

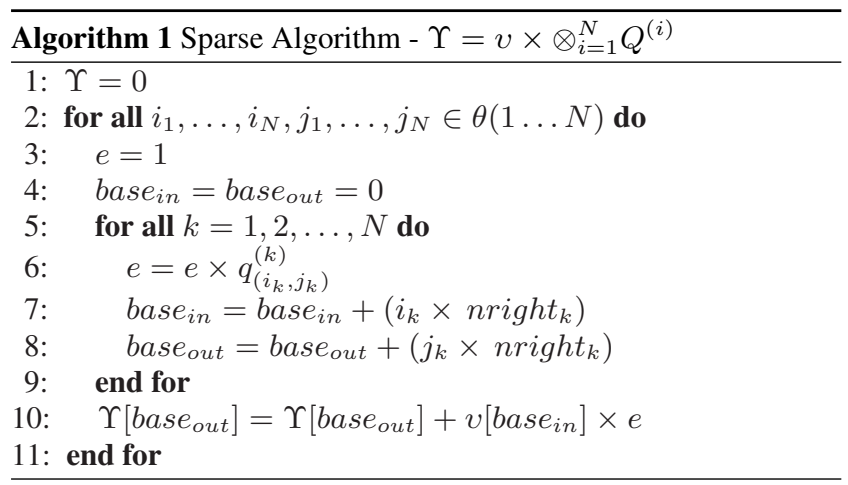

One possible implementation of the sparse algorithm is presented above. The number of floating point multiplications for this implementation is:

$$
N \times \prod_{i=1}^{N} n z_{i}
$$

However, in this version, all nonzero elements of $Q$ are generated during the algorithm execution. Such elements generation represents $(N-1) \times \prod_{i=1}^{N} n z_{i}$ multiplications that could be avoided by generating one (usually huge) sparse matrix to store these $\prod_{i=1}^{N} n z_{i}$ nonzero elements. It would eliminate rows 3 and 6 from the Sparse algorithm and reduce the number of floating point multiplications to just:

$$
\prod_{i=1}^{N} n z_{i}
$$

This option allows the Sparse algorithm to be very time-efficient, but potentially very memory demanding due to the storage of a, potentially huge, sparse matrix $Q$.

Another interesting approach to the sparse algorithm is to keep the nonzero elements inside the algorithm, but factorizing previous calculations [8]. Note that all combinations of elements of each matrix of the tensor product have multiplications in common, i.e., the combinations can be generated considering their partial results referred to the others. Such solution reduces the complexity in terms of number of multiplications applying an algorithm to exploit levels of factoring reusing previous calculations. This approach is, nevertheless, very sensitive to the number of functional elements in the tensor product term and may not produce good results. Anyway, the worst case scenario of this variant can bring back the number of required multiplications to those stated in Equation 2, and the best case (no functional elements at all) will give:

$$
\left(\sum_{i=1}^{N} \prod_{j=1}^{i} n_{i}\right)+\prod_{i=1}^{N} n z_{i}
$$


The sparse approach considered will be the more time-efficient version, i.e., the variant with the previous generation and storage of nonzero elements of $Q$. Such variant demands the smaller number of floating point multiplications (Equation 3), but demands the storage of a sparse matrix with $\prod_{i=1}^{N} n z_{i}$ nonzero elements.

\section{MEMORY-EFFICIENT ALGORITHM}

The basic principle of the Shuffle method is the application of the decomposition of a tensor product in the ordinary product of normal factors property [16]:

$$
\begin{aligned}
Q^{(1)} \otimes & Q^{(2)} \otimes \ldots \otimes Q^{(N-1)} \otimes Q^{(N)}= \\
& \left(Q^{(1)} \otimes I_{n_{2}} \otimes \ldots \otimes I_{n_{N-1}} \otimes I_{n_{N}}\right) \times \\
& \left(I_{n_{1}} \otimes Q^{(2)} \otimes \ldots \otimes I_{n_{N-1}} \otimes I_{n_{N}}\right) \times \\
& \ldots \\
& \left(I_{n_{1}} \otimes I_{n_{2}} \otimes \ldots \otimes Q^{(N-1)} \otimes I_{n_{N}}\right) \times \\
& \left(I_{n_{1}} \otimes I_{n_{2}} \otimes \ldots \otimes I_{n_{N-1}} \otimes Q^{(N)}\right)
\end{aligned}
$$

Hence, the Shuffle algorithm consists in multiplying successively a vector by each normal factor. More precisely, vector $v$ is multiplied by the first normal factor, then the resulting vector is multiplied by the next one, and so on, until the last. These multiplications are done using small vectors called $z_{i n}$ and $z_{\text {out }}$ in the Algorithm 2 . These small vectors store the values of $v$ to multiply by the $i^{\text {th }}$ matrix of the normal factor, and store the result, respectively. Their size are given for the value $n r i g h t_{i}$ of the related matrix. Finally, vector $\Upsilon$ stores the results.

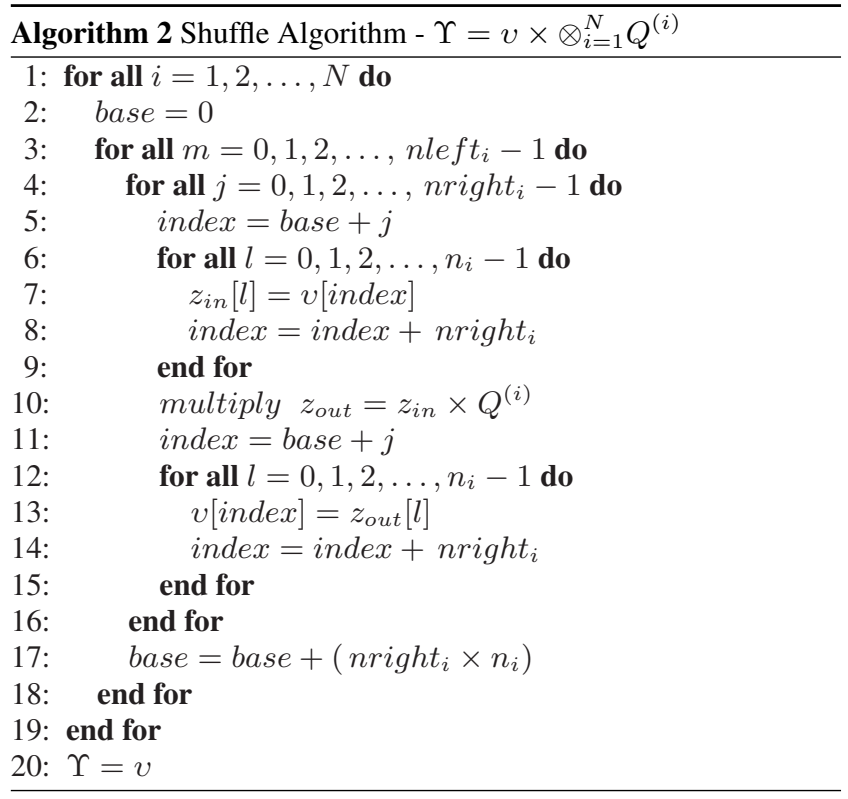

The multiplication of the last normal factor $\left(I_{\text {nleft }_{N}} \otimes Q^{(N)}\right)$ for example, requires the multiplication of contiguous slices of vector $v$, because the $z_{i n}$ vectors are easily located in the product state space, and this normal factor corresponds to $Q^{(N)}$ matrices in the diagonal blocks. A more complicated process to locate the $z_{i n}$ and $z_{\text {out }}$ vectors is required to multiply the other normal factors $\left(I_{\text {nleft }_{i}} \otimes Q^{(i)} \otimes I_{\text {nright }_{i}}\right)$, i.e., the normal factors where the most inner blocks are diagonal matrices. Considering the first normal factor $\left(Q^{(1)} \otimes I_{\text {nright }_{1}}\right)$, i.e., the normal factor composed by $\left(n_{1}\right)^{2}$ blocks containing diagonal matrices with the same element of matrix $Q^{(1)}$, can be treated by assembling the $z_{i n}$ vectors picking vector elements with a $n_{\text {right }_{1}}$ interval.
Generalizing this process for all normal factors, the multiplication of a vector $v$ by the $i^{t h}$ normal factor consists in shuffling the elements of $v$ in order to assemble nle ft $_{i} \times$ nright $_{i}$ vectors of size $n_{i}$ and multiply them by matrix $Q^{(i)}$. Thus, assuming that matrix $Q^{(i)}$ is stored as a sparse matrix, the number of operations needed to multiply a vector by the $i^{\text {th }}$ normal factor is: $n l e f t_{i} \times n r i g h t_{i} \times n z_{i}$, where $n z_{i}$ corresponds to the number of nonzero elements of the $i^{t h}$ matrix of the tensor product term $\left(Q^{(i)}\right)$.

Considering the number of multiplications to all normal factors of a tensor product term, the Shuffle computational cost to perform the basic operation (multiplication of a vector by a tensor product) is given by [16]:

$$
\sum_{i=1}^{N} \text { nleft }_{i} \times \text { nright }_{i} \times n z_{i}=\prod_{i=1}^{N} n_{i} \times \sum_{i=1}^{N} \frac{n z_{i}}{n_{i}}
$$

Another feature of Shuffle algorithm is the optimization for functional elements, i.e., the use of generalized tensor algebra properties and matrices reordering that were already studied in [16]. All those optimizations are very important to reduce the overhead of evaluating functional elements, but such considerations are out of the scope of this paper.

\section{HYBRID METHODS}

Preliminary studies originated the Slice algorithm [17] which is a first approach that takes advantage of the Additive Decomposition property and the decomposition of a tensor product into an ordinary product of normal factors, the basis of a Shuffle-like algorithm. The Additive decomposition property is quite simple and it states that any tensor product can be decomposed into an ordinary sum of hypersparse matrices [8], and for this property the matrices have all elements but one equal to zero. Assuming $\hat{q}_{\left(i_{1}, \ldots, i_{N-1}, j_{1}, \ldots, j_{N}\right)}$ the hypersparse matrix of order $\prod_{i=1}^{N} n_{i}$ composed by only one nonzero element which is in position $i_{1}, \ldots, i_{N}, j_{1}, \ldots, j_{N}$ and it is equal to $\prod_{k=1}^{N} q_{i_{k}, j_{k}}^{(k)}$, the additive decomposition property can be stated as:

$$
\begin{aligned}
Q^{(1)} \otimes Q^{(2)} \otimes \ldots \otimes Q^{(N-1)} \otimes & Q^{(N)}= \\
\sum_{i_{1}=1}^{n_{1}} \ldots \sum_{i_{N}=1}^{n_{N}} \sum_{j_{1}=1}^{n_{1}} \ldots \sum_{j_{N}=1}^{n_{N}} & \\
& \left(\hat{q}_{\left(i_{1}, j_{1}\right)}^{(1)} \otimes \ldots \otimes \hat{q}_{\left(i_{N}, j_{N}\right)}^{(N)}\right)
\end{aligned}
$$

where $\hat{q}_{(i, j)}^{(k)}$ is a hypersparse matrix of order $n_{k}$ in which the element in row $i$ and column $j$ is $q_{i, j}^{(k)}$.

The Algorithm 3 proposes the use of this property decomposing the first $N-1$ matrices of each tensor product, then performing the shuffling operation with the last matrix, for each possible $\hat{q}_{(i, j)}^{(k)}$ special matrix of the term. The new factor created is called $A d$ ditive Unitary Normal Factor (AUNF). It is clear that the nonzero elements generation follows the principles of the Sparse algorithm, and also can use any optimization technique [9]. For each nonzero element $e$, it picks a slice of the vector $v$ (named as $v_{i n}$ ), according to the row position of $e$, and multiply all elements of $v_{i n}$ by element $e$. The resulting vector $v_{i n}$ is multiplied by the last matrix $Q^{(N)}$ accumulating the result $v_{\text {out }}$ in the positions of vector $\Upsilon$ corresponding to the column position of element $e$. The computational cost in multiplications is given by:

$$
\left(\prod_{i=1}^{N-1} n z_{i}\right) \times\left[(N-2)+\left(n_{N}\right)+\left(n z_{N}\right)\right]
$$




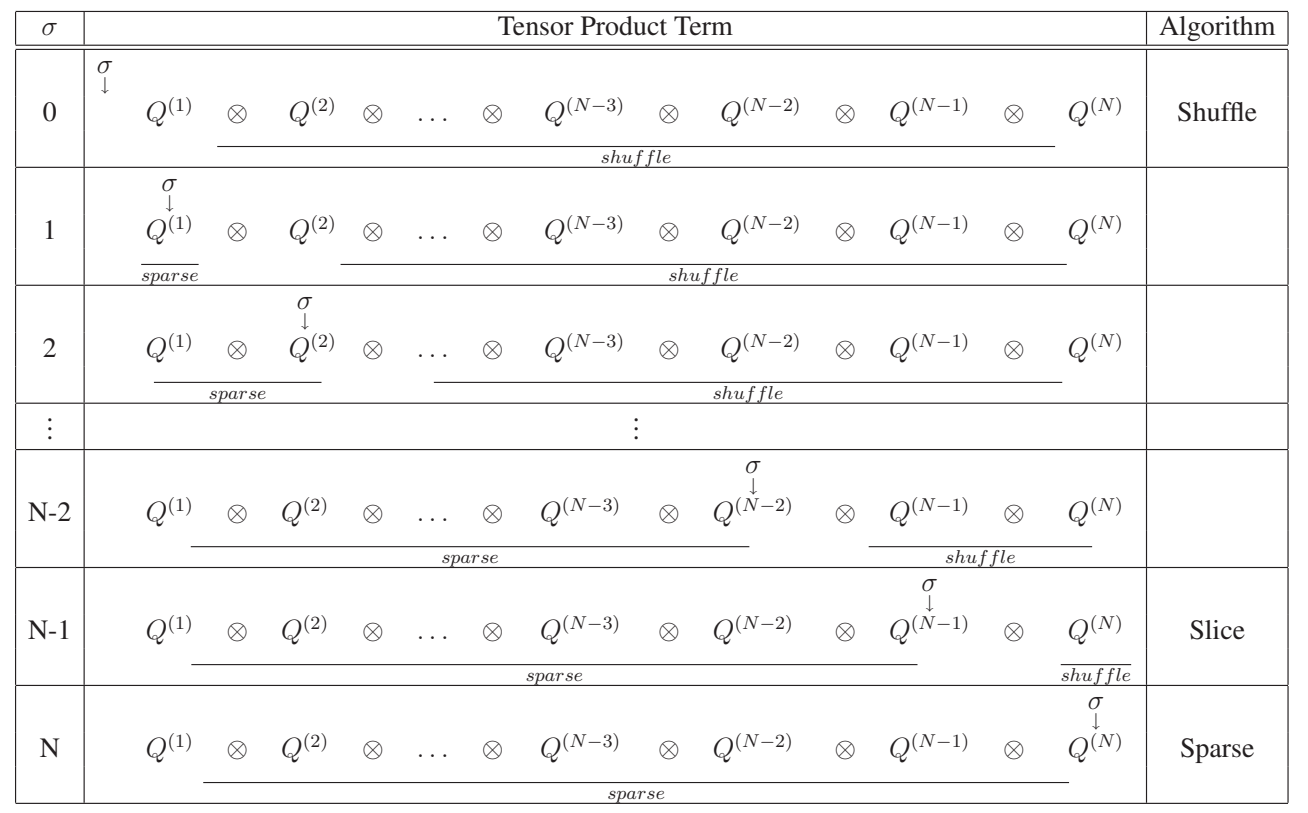

Table 1: Split approach as a generalization of traditional algorithms

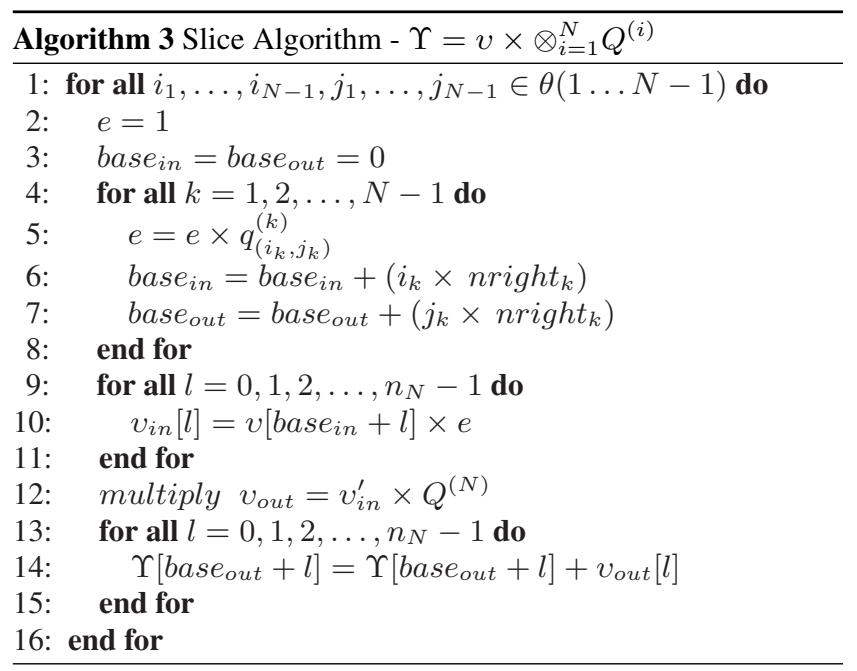

where $\prod_{i=1}^{N-1} n z_{i}$ is the number of $A U N F$ to be generated. For each one, we have $(N-2)$ multiplications needed to its generation (considering the total of $(N-1)$ matrices in the tensor product). This scalar is multiplied by the vector $n_{N}$ times, and then the Shuffle is done with the last matrix costing $n z_{N}$ multiplications.

More details of the Slice algorithm can be found in [17], and even though as a preliminary version of an hybrid numerical method, the Slice algorithm has shown better overall performance than the traditional Shuffle algorithm for some practical SAN examples [18]. But due to its fixed structure, it is not adequate for all models. However, it already takes advantage of the Additive Decomposition property, as a better way to perform the vector-descriptor product in parallel implementations, due the independence among the normal factors generated. The reader interested in parallel versions of solvers for Markovian models may consult [6] and [4].

\section{SPLIT ALGORITHM}

SAN models of practical applications are naturally sparse, the local part of a descriptor is intrinsically very sparse due to the tensor sum structure. The synchronizing events are mostly used to describe exceptional behaviors, therefore it lets this part of the descriptor also quite sparse. Hence, following the Slice algorithm guidelines, each tensor product of matrices can be partitioned in two different groups: the first one with the sparser matrices; and the second one with the matrices with a larger number of nonzero elements. A Sparse-like approach could be applied to the first group of $K$ matrices generating $A U N F$, as Slice does with the first $N-1$ matrices. Each one of those AUNF should be tensorly multiplied by the second group of matrices using a Shuffle-like approach (Slice deals only with the last matrix in this part). Considering that, the idea is to split the tensor terms in two sets of matrices treating them in two different ways, with a certain degree of dependence. Due to this, the novel algorithm called Split is also a generalization for Slice algorithm, because it follows the idea of having distinct parts to treat.

Table 1 presents the general idea of the Split algorithm graphically. It is important to notice that the index of the matrix chosen for delimiting the end of the first set is assigned to the cut-parameter $\sigma$ separating the first $K$ matrices. It is possible to observe that the Sparse $(\sigma=N)$, the Slice $(\sigma=N-1)$ and the Shuffle $(\sigma=0)$ methods are also particular cases of the Split algorithm, i.e., they are also possibilities of splitting in a tensor term composed by $N$ matrices.

Algorithm 4 defines formally the steps of the Split approach. It consists in the computation of the element $e$ of each AUNF by multiplying one nonzero element of each matrix of the first set of matrices (from $Q^{(1)}$ to $Q^{(\sigma)}$ ). According to the elements row indexes used to generate element $e$, a contiguous slice of input vector $v$ is taken $\left(v_{i n}\right)$. Vector $v_{i n}$ of size nright $_{\sigma}$ (corresponding to the product of the order of all matrices after the cut-parameter $\sigma$ of the tensor product term) is multiplied by element $e$. The resulting vector $\left(v_{i n}^{\prime}\right)$ is used as input vector to the Shuffle-like multiplication 
by the tensor product of the matrices in the second set of matrices (from $Q^{(\sigma+1)}$ to $Q^{(N)}$ ), due to this there is a kind of dependency between both sets.

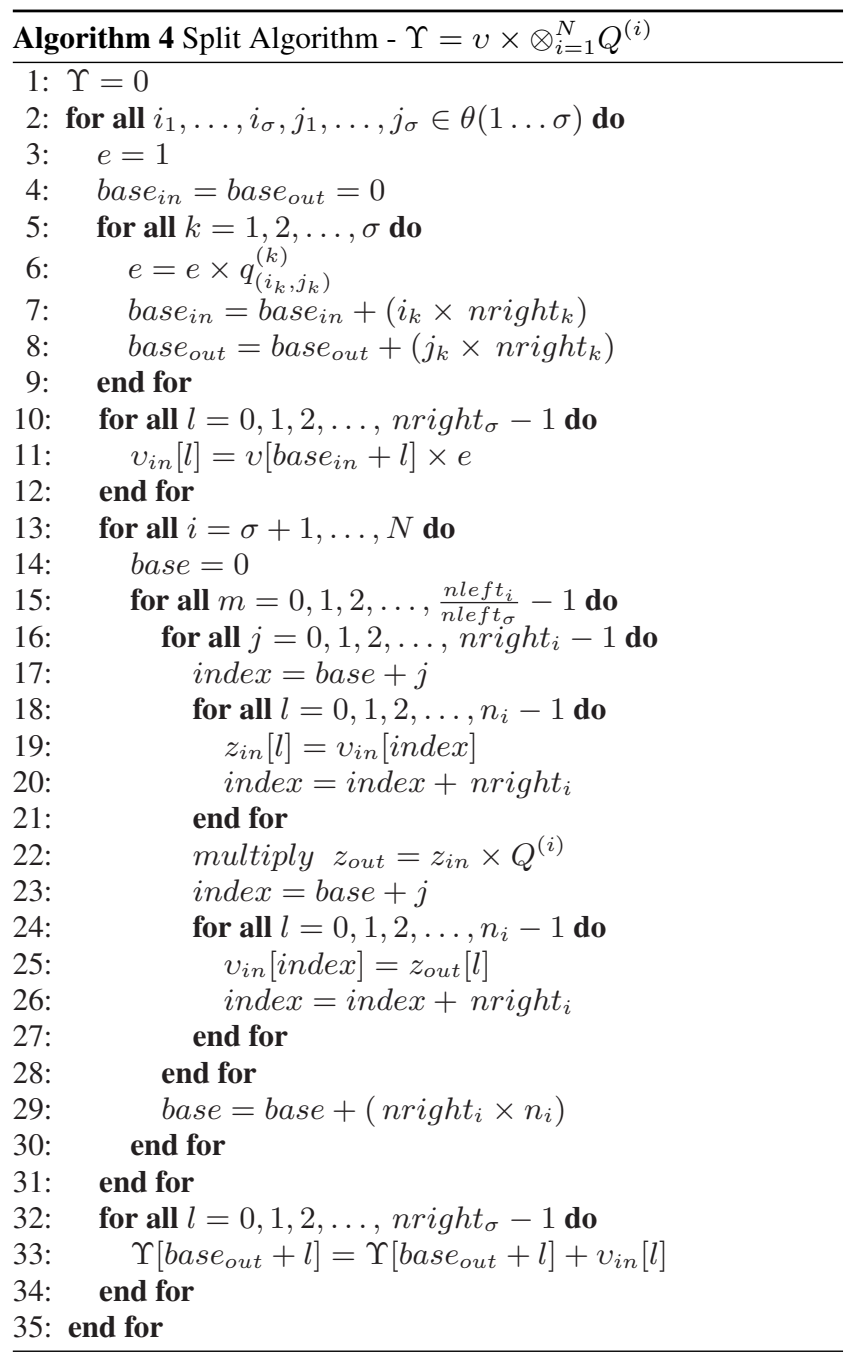

The computational cost in number of multiplications (Equation 9) for the Split algorithm is computed taking into account the number of multiplications performed to generate the nonzero element of each AUNF $(\sigma-1)$, plus the number of multiplications of the scalar by each position value of the vector $v_{i n}$. There is also the cost to multiply the values in the input vector $v_{i n}^{\prime}$ by the tensor product of matrices in the Shuffle-like part.

$$
\left(\prod_{i=1}^{\sigma} n z_{i}\right)\left[(\sigma-1)+\left(\prod_{i=\sigma+1}^{N} n_{i}\right)+\left(\prod_{\sigma+1}^{N} n_{i} \times \sum_{i=\sigma+1}^{N} \frac{n z_{i}}{n_{i}}\right)\right]
$$

In practical implementations of vector-descriptor multiplication algorithms, improvements can be done to speedup the execution. These optimizations can change significantly the theoretical computational cost presented in the Equation 9.

Regarding the Shuffle algorithm, there is an optimization on the way of handling identity matrices. Those matrices do not need to generate normal factors, since being identity matrices, they generate a normal factor that is also an (huge) identity matrix itself. The computational cost is clearly reduced in the Shuffle algorithm execution when using this solution. It corresponds to transform the number of floating point multiplications equation for the Shuffle algorithm (Equation 6) to:

$$
\prod_{i=1}^{N} n_{i} \times \sum_{\substack{i=1 \\ i f f Q^{(i)} \neq I d}}^{N} \frac{n z_{i}}{n_{i}}
$$

This improvement suggests the same skipping-identities optimization to the Shuffle-like part (matrices $Q^{(\sigma+1)}$ to $Q^{(N)}$ ) of the Split algorithm (4) identifying if the matrix indexed by variable $i$ of the algorithm $\left(Q^{(i)}\right)$ is not an identity matrix, adding to the cost $\frac{n z_{i}}{n_{i}}$ multiplications only for these ones. Analogously to Shuffle algorithm, Equation 9 will be rewritten changing the shuffle-part cost accordingly. The resulting number of floating point multiplications for the Split algorithm will be:

$$
\left(\prod_{i=1}^{\sigma} n z_{i}\right)\left[(\sigma-1)+\left(\prod_{i=\sigma+1}^{N} n_{i}\right)+\left(\prod_{\sigma+1}^{N} n_{i} \times \sum_{\substack{i=\sigma+1 \\ i f f Q^{(i)} \neq I d}}^{N} \frac{n z_{i}}{n_{i}}\right)\right]
$$

Usually the tensor product terms of a SAN model are very sparse (a few thousands nonzero elements). The only cases where a more significant number of nonzero elements is found are those when we are dealing with a tensor product term with many identity matrices. It is important to recall that to each $A U N F$ scalar $e$ is computed as the product of one single element of each matrix. The second optimization is the precomputation of these nonzero elements and their storage consequently, that for pure sparse solving approaches, this optimization was already largely studied [9]. It results in a reduction of the Split algorithm computational cost similar to that one presented in Section 2 regarding the computation of nonzero elements. Hence, the final definition of the number of floating point multiplications for the Split algorithm is no longer defined by Equation 11, but as:

$$
\left(\prod_{i=1}^{\sigma} n z_{i}\right)\left[\left(\prod_{i=\sigma+1}^{N} n_{i}\right)+\left(\prod_{\sigma+1}^{N} n_{i} \times \sum_{\substack{i=\sigma+1 \\ i f f Q^{(i)} \neq I d}}^{N} \frac{n z_{i}}{n_{i}}\right)\right]
$$

Note that it is not surprising if for very sparse tensor products the best Split option was the pure Sparse approach which, obviously, is much more effective if the nonzero elements do not have to be recomputed at each vector multiplication but it means that we need to store a possibly huge matrix. Therefore, the Split choice must also balance the computational cost in terms of multiplications and memory needs.

\section{NUMERICAL RESULTS}

The experiments results were collected running a prototype of traditional methods and the new one, the Split algorithm, on a 3.2 $\mathrm{GHz}$ Intel Xeon under Linux operating system with 4 GBytes of memory. The prototype was compiled using $g++$ compiler with optimizations $(-O 3)$. The methods were executed for practical examples of SAN models [13, 3], running all tensor product terms in all possible cut-parameters, collecting outputs for 100 runs. The results were obtained in time intervals with $95 \%$ of confidence.

The presented results for Split algorithm executions are the collection of the best execution times obtained given the confidence interval. It also considers different cut-parameters $\sigma$ to each tensor product term in the descriptor. However, we consider a fix order of automata and no automata permutations were considered. In such way, it is possible to say that the Split results presented here tend to force the trade-off between time and memory efficiency towards 


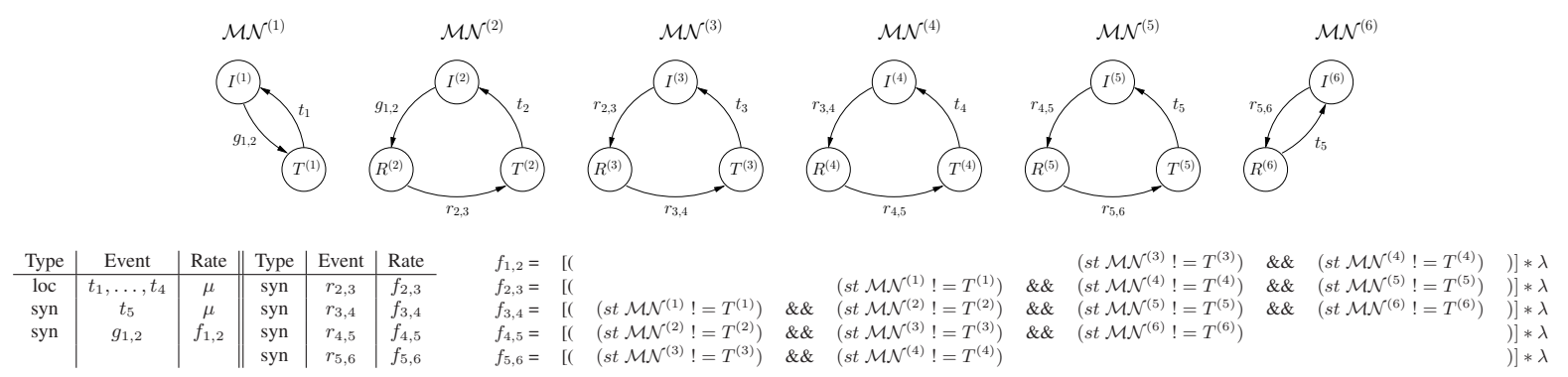

Figure 1: Ad Hoc WSN SAN Model (6 nodes)

\begin{tabular}{|c|c|c|c|c|c|c|c|c|c|c|}
\hline \multirow[b]{2}{*}{$N$} & \multirow[b]{2}{*}{ PSS } & \multicolumn{3}{|c|}{ Shuffle } & \multicolumn{3}{|c|}{ Split } & \multicolumn{3}{|c|}{ Sparse } \\
\hline & & time $(\mathrm{s})$ & size $(\mathrm{Kb})$ & fpm (Eq. 10) & time (s) & size $(\mathrm{Kb})$ & fpm (Eq. 12) & time $(\mathrm{s})$ & size $(\mathrm{Kb})$ & fpm (Eq. 3) \\
\hline 6 & 324 & 0.00075 & 1.52 & 7,344 & 0.00013 & 5.30 & 1,798 & 0.00025 & 18.93 & 1,114 \\
\hline 8 & 2,916 & 0.00633 & 2.25 & 93,312 & 0.00071 & 103.22 & 14,332 & 0.00163 & 219.88 & 13,928 \\
\hline 10 & 26,244 & 0.07454 & 2.99 & $1,084,752$ & 0.01307 & 170.11 & 185,364 & 0.01520 & $2,510.61$ & 160,488 \\
\hline 12 & 236,196 & 0.87020 & 3.72 & $11,967,264$ & 0.18774 & $1,461.79$ & $2,230,740$ & 0.20951 & $27,513.35$ & $1,760,616$ \\
\hline 14 & $2,125,760$ & 9.35304 & 4.46 & $127,545,900$ & 1.75147 & $13,536.58$ & $22,674,856$ & 2.07020 & $292,060.08$ & $18,691,560$ \\
\hline 16 & $19,131,900$ & 96.44355 & 5.19 & $1,326,477,700$ & 17.99368 & $118,103.25$ & $235,251,512$ & 21.02443 & $3,028,726.82$ & $193,838,184$ \\
\hline
\end{tabular}

Table 2: Ad Hoc WSN Model Results

time savings. It would be absurd to take the opposite decision, since forcing Split to save memory would result in practically the same efficiency as the Shuffle algorithm, which already gives the more memory-efficient solution.

\subsection{Ad Hoc WSN model}

The SAN model in the Figure 1 represents a chain of mobile nodes in a Wireless Sensor Network (Ad Hoc WSN model) running over the 802.11 standard for ad hoc networks. This model [13] resembles the ad hoc forwarding experiment presented in [20] using $\mathrm{SAN}$. The chain of $N$ nodes modeled, have the first node $\mathcal{M} \mathcal{N}^{(1)}$ (Source automaton) that generates packets as fast as the standard allows. The packets are forwarded through the chain by the Relay automata called $\mathcal{M N} \mathcal{N}^{(i)}$, where the variable $i$ is among the value 2 and $(N-1)$, to the last node $\mathcal{M N}^{(N)}$ (Sink automaton).

This SAN model family generically has $(N-2)$ local events and $N$ synchronizing events among $N$ automata. The Descriptor $Q$ for these models is formed by a set of $[(N-2)+2 N]$ tensor product terms. The numerical results were obtained for this model extending the number of automata and, consequently, the number of tensor product terms.

Table 2 shows the results for the three methods, Shuffle, Split and Sparse, divided each in three columns representing time in seconds (per iteration), size in KBytes $(\mathrm{Kb})$ and the computational cost in floating point multiplications (fpm). The column PSS stands for the product state space $\prod_{i=1}^{N} n_{i}$, which is the Cartesian product of the automata orders (the size of the probability vector). As the number of nodes in the mobile chain increases $(N=6,8,10,12,14,16)$, so does the time to solve as well as the memory needed. Since Shuffle is memory-efficient, it is slower than the other two methods. These methods, on the contrary, are much more memory consuming, to pay for the time efficiency.

For small models ( $N<12$ nodes) the time and memory efficiency are reasonable enough to be dealt regardless of algorithm in virtually any machine. It demands few more the 2 Mbytes in the sparse approach and it takes less than 100 milliseconds per iteration for all algorithms. However, even in this small examples we notice the quite impressive memory efficiency of the Shuffle al- gorithm that keeps the memory needs insignificant even for quite large models.

The remarkable result in Table 2 is the better time efficiency that beats even the sparse approach. Although, for each tensor product, sparse approach could be faster for most cases, terms with many identity matrices could have a better time efficiency in Shuffle or Split algorithms. Since a SAN model is composed of many tensor product terms, Split will be the best option in product terms where the sparse approach could be faster, but too memory demanding.

This is clearly the case of the largest model $(N=16)$ where Split is around 3 seconds faster than Sparse with a memory need of little more than 100 Mbytes, rather than 3 Gbytes needed by the sparse solution, i.e., Split takes for this case almost 30 times less memory and still improves the time efficiency compared to Sparse. It is important to observe, as well, that this model has a considerable product state space of more than 19 million states. Such large model could be nearly intractable if a time and memory efficient solution is not found.

Note that the number of fpm computed to each algorithm is not relevant to indicate a better performance in time. This behavior needs more observation, but, approximatively, we dare to say that other indications like unconsidered operations or maybe allocations/deallocations may influence the performance of the algorithms.

\subsection{Master-Slave Parallel Model}

This SAN model in the Figure 2 refers to an evaluation of the master-slave parallel implementation of the Propagation algorithm considering asynchronous communication [3], indicating to parallel program developers what are the possible execution bottlenecks before the implementation. This model contains one Master automaton, one huge Buffer automaton, and $S$ automata Slave ${ }^{(i)}$, where $i=1 \ldots S$.

The total number of automata is generically given by $(S+2)$, having $S$ local events and $(3 S-3)$ synchronizing events. The Descriptor $Q$ in this case, is formed by a set of $(7 S-8)$ tensor terms. This model was extended to run for different numbers of slaves $(S=3,4,5,6,7,8,10,12)$ and the buffer is of forty po- 


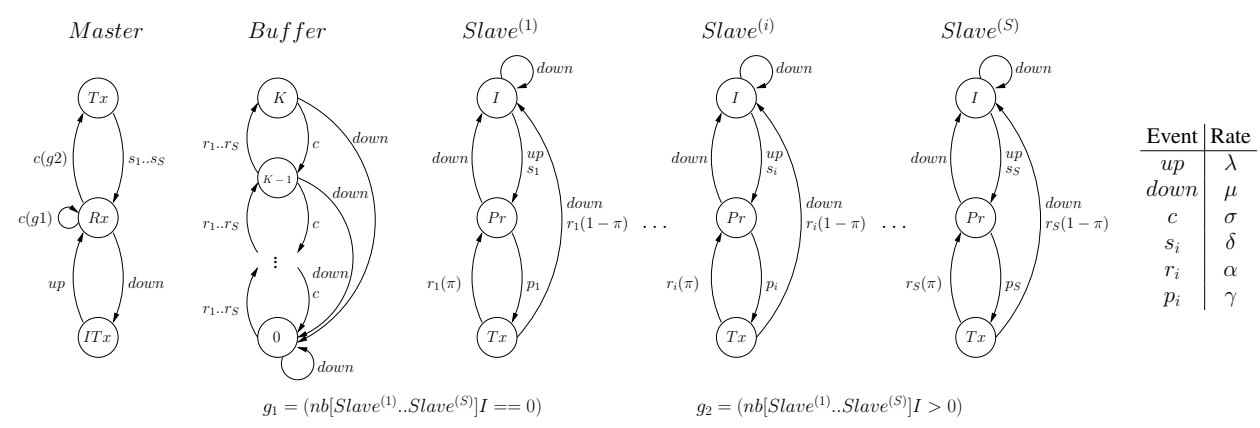

Figure 2: Master-Slave Parallel SAN Model

\begin{tabular}{|c|c|c|c|c|c|c|c|c|c|c|}
\hline \multirow[b]{2}{*}{$S$} & \multirow[b]{2}{*}{ PSS } & \multicolumn{3}{|c|}{ Shuffle } & \multicolumn{3}{|c|}{ Split } & \multicolumn{3}{|c|}{ Sparse } \\
\hline & & time $(\mathrm{s})$ & size $(\mathrm{Kb})$ & fpm (Eq. 10) & time $(\mathrm{s})$ & size $(\mathrm{Kb})$ & fpm (Eq. 12) & time $(\mathrm{s})$ & size $(\mathrm{Kb})$ & fpm (Eq. 3) \\
\hline 3 & 3,321 & 0.00932 & 11.19 & 128,385 & 0.00174 & 175.11 & 37,902 & 0.00257 & 381.07 & 23,672 \\
\hline 4 & 9,963 & 0.02884 & 14.23 & 497,097 & 0.00711 & 508.61 & 116,780 & 0.00869 & $1,435.29$ & 90,948 \\
\hline 5 & 29,889 & 0.09830 & 16.63 & $1,797,228$ & 0.02231 & $1,447.69$ & 399,036 & 0.02537 & $5,229.25$ & 333,608 \\
\hline 6 & 89,667 & 0.35073 & 20.31 & $6,488,829$ & 0.09305 & $3,183.29$ & $1,742,271$ & 0.10873 & $18,531.62$ & $1,184,724$ \\
\hline 7 & 269,001 & 1.31777 & 23.35 & $22,488,916$ & 0.37132 & $9,446.93$ & $6,555,978$ & 0.40385 & $64,222.72$ & $4,108,760$ \\
\hline 8 & 807,003 & 4.58053 & 26.43 & $76,534,019$ & 1.23928 & $28,236.07$ & $23,037,480$ & 1.34866 & $231,315.37$ & $14,802,495$ \\
\hline 10 & $7,263,030$ & 50.07517 & 32.43 & $847,176,190$ & 12.92194 & $240,596.27$ & $246,651,139$ & 13.90858 & $2,363,273.71$ & $151,247,442$ \\
\hline 12 & $65,367,200$ & 535.28774 & 38.54 & $9,137,063,300$ & 135.94263 & $2,282,495.12$ & $2,787,370,431$ & 147.59428 & $26,195,236.61$ & $1,676,492,676$ \\
\hline
\end{tabular}

Table 3: Master-Slave Parallel Model Results

sitions $(K=40)$. Table 3 shows the results for all these model extensions.

The results are consistent with those obtained in the previous section. Split once again demonstrate a better time efficiency. In fact it presents, in general, results a little faster than the sparse approach, i.e., roughly around $10 \%$ faster in the large models.

However, the memory savings obtained in this second set of examples seem less impressive than those obtained for the Ad Hoc WSN models. Split still gives a considerable reduction for the huge last example $(S=12)$ bringing the memory needs from nearly intractable 26 Gbytes in sparse approach to large, but tractable 2.2 Gbytes. Once again, it is important to keep in mind that we are dealing with a model with 65 million states so significant amount of memory and time is expected to achieve a stationary or transient solution. An interesting point, still subject to deeper analysis, is to observe how inadequate is the number of fpm to predict how fast an algorithm will execute.

\section{CONCLUSION}

The main contribution of this paper is the proposition of a flexible hybrid vector-descriptor algorithm. Applying the proposed algorithm to SAN models of real problems verifies the good trade-off between memory and time efficiency of the Split algorithm when compared to traditional Sparse and Shuffle approaches. Considering that we need many iterations to calculate the final probability vector, the memory and time spent tradeoffs surely should be evaluated and balanced, maybe according to the available time and computational resources. Nevertheless, it is also shown that the Split algorithm is flexible enough to deliver in extreme cases at least the same time efficiency as the sparse approach, or, alternatively, the same memory efficiency as the shuffle approach.

Since tensor terms can be formed differently due the different structured models we deal with, the performance can also be very dependent on the choice of matrices placed in each group. The tensor product terms that do not have too many identity matrices, or no identities at all, can be multiplied in a sparse fashion. However, Shuffle deals better with terms containing many identities because it simply jumps the execution for the next matrix to multiply. Our experience with structured models suggests that these tensor product terms, with a reasonable number of identity matrices, are the most commonly encountered ones, but if the memory available is not a problem, it is better to treat them in a sparse manner as much as possible.

A clearly open problem is the choice of division point in each tensor term (choice of cut-parameter $\sigma$ ) and, even more important, the choice of order for terms. However, the research for an heuristic to automatically choose the best order of matrices and the cut-parameter, for each tensor product is a considerable research challenge. This is not a trivial task, due to the tensor product term formation and intrinsic matrices details such as order, total nonzero elements and computational cost in multiplications. These parameters opens the possibility of a thorough analysis of the related theoretical computational cost. Another important aspect is the fact that we can have a tradeoff between memory usage and time spent, i.e., if one have lots of memory and wants performance, the cutparameter could be more easily shifted to use the sparse approach. If memory is limited, the cut-parameter should allow more shuffle operations.

Additionally, the proposed Split algorithm could be enhanced with considerations about the impact of functional elements (with their particular dependencies) in the descriptor. A similar work about these functional dependency changed completely the performance of the Shuffle algorithm [16]. It is only natural to estimate that similar gains with functional dependencies analysis and possible automata permutations could benefit the Split algorithm as well.

Finally, it is also possible to foresee an even more complex analysis that considers not only a sequential version of the Split algorithm, but also parallel implementations. For the sequential version, memory and time efficiency are dealt as a single demand, but 
parallel implementations should consider the amount of memory needed, volume of data exchanged and processing demands to be as evenly as possible distributed among parallel machines. Obviously, this further analysis is much more deep and complex since neither the number of floating point multiplications, nor any other known index for that matter, seems to be a good estimation of processing time (see the previous section remarks). Nevertheless, it still seems an interesting field of future research.

We know for a fact that the Split algorithm can be subject to considerable improvements in a near future. Despite that, a not very thorough analysis were done for the presented examples, choosing other automata orders and cut-parameters $\sigma$. Preliminary results for the Split sequential implementation shows quite impressive gains in terms of processing time.

\section{REFERENCES}

[1] M. Ajmone-Marsan, G. Conte, and G. Balbo. A Class of Generalized Stochastic Petri Nets for the Performance Evaluation of Multiprocessor Systems. ACM Transactions on Computer Systems, 2(2):93-122, 1984.

[2] V. Amoia, G. D. Micheli, and M. Santomauro. Computer-Oriented Formulation of Transition-Rate Matrices via Kronecker Algebra. IEEE Transactions on Reliability, R-30(2):123-132, 1981.

[3] L. Baldo, L. Brenner, L. G. Fernandes, P. Fernandes, and A. Sales. Performance Models for Master/Slave Parallel Programs. Electronic Notes In Theoretical Computer Science, 128(4):101-121, April 2005.

[4] L. Baldo, L. G. Fernandes, P. Roisenberg, P. Velho, and T. Webber. Parallel PEPS Tool Performance Analysis using Stochastic Automata Networks. In Euro-Par 2004 International Conference on Parallel Processing, volume 3149 of $L N C S$, pages 214-219, Pisa, Italy, 2004.

[5] L. Brenner, P. Fernandes, and A. Sales. The Need for and the Advantages of Generalized Tensor Algebra for Kronecker Structured Representations. International Journal of Simulation: Systems, Science \& Technology, 6(3-4):52-60, February 2005.

[6] P. Buchholz. A distributed numerical/simulative algorithm for the analysis of large continuous time markov chains. In PADS '97: Proceedings of the eleventh Workshop on Parallel and Distributed Simulation, pages 4-11, USA, 1997.

[7] P. Buchholz. A new approach combining simulation and randomization for the analysis of large continuous time Markov Chains. ACM Trans. Model. Comput. Simul., 8(2):194-222, 1998

[8] P. Buchholz, G. Ciardo, S. Donatelli, and P. Kemper. Complexity of memory-efficient Kronecker operations with applications to the solution of Markov models. INFORMS Journal on Computing, 13(3):203-222, 2000.

[9] P. Buchholz, G. Ciardo, S. Donatelli, and P. Kemper. Complexity of memory-efficient kronecker operations with applications to the solution of markov models. INFORMS Journal on Computing, 12(3):203-222, 2000.

[10] P. Buchholz and P. Kemper. Hierarchical reachability graph generation for Petri nets. Formal Methods in Systems Design, 21(3):281-315, 2002.

[11] M. Davio. Kronecker Products and Shuffle Algebra. IEEE Transactions on Computers, C-30(2):116-125, 1981.

[12] S. Donatelli. Superposed generalized stochastic Petri nets: definition and efficient solution. In Proceedings of the $15^{\text {th }}$ International Conference on Applications and Theory of
Petri Nets, pages 258-277, 1994.

[13] F. L. Dotti, P. Fernandes, A. Sales, and O. M. Santos. Modular Analytical Performance Models for Ad Hoc Wireless Networks. In $3^{\text {rd }}$ International Symposium on Modeling and Optimization in Mobile, Ad Hoc, and Wireless Networks, pages 164-173, Trentino, Italy, April 2005.

[14] F. L. Dotti and L. Ribeiro. Specification of Mobile Code Systems using Graph Grammars. In Formal Methods for Open Object-Based Distributed Systems IV, pages 45-63, Stanford, USA, 2000. Kluwer Academic Publishers.

[15] P. Fernandes. Methodes numeriques pour la solution de systhemes Markoviens a grand espace d'etats. $\mathrm{PhD}$ thesis, Institut National Polytechnique de Grenoble, France, 1998.

[16] P. Fernandes, B. Plateau, and W. J. Stewart. Efficient descriptor - Vector multiplication in Stochastic Automata Networks. Journal of the ACM, 45(3):381-414, 1998.

[17] P. Fernandes, R. Presotto, A. Sales, and T. Webber. An Alternative Algorithm to Multiply a Vector by a Kronecker Represented Descriptor. In $21^{\text {st }}$ UK Performance Engineering Workshop, pages 57-67, Newcastle, UK, June 2005.

[18] P. Fernandes, R. Presotto, A. Sales, and T. Webber. An Alternative Algorithm to Multiply a Vector by a Kronecker Represented Descriptor. Technical Report TR 047, PUCRS, Porto Alegre, 2005. http://www.inf.pucrs.br/tr/tr047.pdf.

[19] J. Hillston and L. Kloul. An Efficient Kronecker Representation for PEPA models. In Proceedings of the first joint PAPM-PROBMIV Workshop), pages 120-135, Aachen, Germany, September 2001.

[20] J. Li, C. Blake, D. S. J. D. Couto, H. I. Lee, and R. Morris. Capacity of Ad Hoc Wireless Networks. In $7^{\text {th }}$ Annual International Conference on Mobile Computing and Networking, pages 61-69, Rome, Italy, July 2001.

[21] A. S. Miner. Efficient solution of GSPNs using Canonical Matrix Diagrams. In $9^{\text {th }}$ International Workshop on Petri Nets and Performance Models (PNPM'01), pages 101-110, Aachen, Germany, September 2001.

[22] A. S. Miner and G. Ciardo. A data structure for the efficient Kronecker solution of GSPNs. In Proceedings of the $8^{\text {th }}$ International Workshop on Petri Nets and Performance Models, pages 22-31, Zaragoza, Spain, September 1999.

[23] A. S. Miner and G. Ciardo. Efficient Reachability Set Generation and Storage Using Decision Diagrams. In Proceedings of the $20^{\text {th }}$ International Conference on Applications and Theory of Petri Nets, volume 1639 of LNCS, pages 6-25, USA, June 1999.

[24] B. Plateau and K. Atif. Stochastic Automata Networks for modelling parallel systems. IEEE Transactions on Software Engineering, 17(10):1093-1108, 1991.

[25] W. J. Stewart. MARCA: Markov chain analyzer. A software package for Markov modeling, pages 37-62. Numerical Solution of Markov Chains. New York, 1991.

[26] W. J. Stewart. Introduction to the numerical solution of Markov chains. Princeton University Press, 1994. 